\title{
Rapid, accurate, multifunctional and self-assisted vision assessment and screening with interactive desktop autostereoscopy
}

\author{
Xiaoke Li ${ }^{1 \#}$, Jing Zhong ${ }^{2 \#}$, Yiyao Wang ${ }^{2 \#}$, Hantao Zhang ${ }^{1}$, Jinrong $\mathrm{Li}^{2}$, Kunyang $\mathrm{Li}^{1}$, Li Gu${ }^{2}, \mathrm{Min} \mathrm{Zheng}^{1}$, \\ Jin Yuan', Hang Fan ${ }^{3}$, Dongyan Deng, Yao Wang ${ }^{3}$, Jianying Zhou ${ }^{1}$ \\ ${ }^{1}$ State Key Laboratory of Optoelectronic Materials and Technology, School of Physics, Sun Yat-sen University, Guangzhou, China; ${ }^{2}$ State Key \\ Laboratory of Ophthalmology, Zhongshan Ophthalmic Center, Zhongshan School of Medicine, Sun Yat-sen University, Guangzhou, China; \\ ${ }^{3}$ Guangzhou Midstereo Co., 514-515 Building B, University Science and Technology Park, Guangzhou, China \\ Contributions: (I) Conception and design: J Zhou, J Yuan; (II) Administrative support: J Zhou, J Yuan; (III) Provision of study materials or patients: J \\ Zhou, J Yuan; (IV) Collection and assembly of data: X Li, J Zhong, Y Wang; (V) Data analysis and interpretation: H Fan, H Zhang, K Li, L Gu, Y \\ Wang, M Zheng; (VI) Manuscript writing: All authors; (VII) Final approval of manuscript: All authors. \\ \#These authors contributed equally to this work. \\ Correspondence to: Jin Yuan. State Key Laboratory of Ophthalmology, Zhongshan Ophthalmic Center, Zhongshan School of Medicine, Sun Yat- \\ sen University, Guangzhou 510064, China. Email: yuanjincornea@126.com; Jianying Zhou. State Key Laboratory of Optoelectronic Materials and \\ Technology, School of Physics, Sun Yat-sen University, Guangzhou 510275, China. Email: stszjy@mail.sysu.edu.cn.
}

Background: This study aimed to develop an interactive vision screening tool based on desktop autostereoscopy and evaluate its feasibility for testing visual acuity, colour vision, stereo vision and binocular balance clinically.

Methods: An interactive desktop autostereoscopy vision test was developed making it remarkably convenient for individuals to undergo multiple visual function assessments in a single test. With this rapid screening process, an individual's visual acuity, colour vision, stereo vision and binocular balance can be assessed within several minutes. A total of 155 healthy subjects were enrolled to compare the clinical repeatability, accuracy, inter-visit variability, likeability and efficiency between the autostereoscopy and traditional method.

Results: In the repeatability test, the visual acuity measured with autostereoscopy was $0.045 \pm 0.018$ and $0.035 \pm 0.018(\mathrm{P}=0.702)$ for the first and second tests, respectively. The mean logarithm of the Minimum Angle of Resolution ( $\log M A R)$ visual acuities measured with the Early Treatment Diabetic Retinopathy Study (EDTRS) chart and autostereoscopy test were $0.04 \pm 0.02$ and $0.05 \pm 0.02$, respectively, which were not significantly different $(\mathrm{P}=0.849)$. The correlation between these two kinds of tests was statistically significant (Spearman correlation coefficient $=0.829, \mathrm{P}<0.001$ ). The results for colour vision, stereo vision, and binocular vision are presented, and the effectiveness of the autostereoscopic method is supported with qualitative data comparing its results with those of the traditional methods. In the likeability test, the EDTRS chart and autostereoscopy test had scores of $2.21 \pm 0.53$ and $3.04 \pm 0.07$, while the traditional and autostereoscopy tests for colour vision, stereo vision, and binocular vision had scores of $2.02 \pm 0.59$ and $3.36 \pm 0.93$, respectively $(\mathrm{P}<0.001)$. Regarding visual fatigue, the mean scores were $0.69 \pm 0.04$ and $0.42 \pm 0.04(\mathrm{P}<0.001)$ with the EDTRS chart and autostereoscopy test, respectively. Regarding work efficiency, the average testing times per person was $59.65 \pm 0.66$ and $48.92 \pm 0.86 \mathrm{~s}(\mathrm{P}<0.001)$ with the EDTRS chart and autostereoscopy test, respectively.

Conclusions: The autostereoscopy test was conclusively shown to be valid, efficient and repeatable for the measurement of visual acuity, colour vision, stereo vision, and binocular vision, and the process was subjectively well-liked and comfortable.

Keywords: Autostereoscopy; interactive desktop; vision assessment 
Submitted Apr 27, 2020. Accepted for publication Aug 30, 2020.

doi: 10.21037/atm-20-3555

View this article at: http://dx.doi.org/10.21037/atm-20-3555

\section{Introduction}

Vision impairment, notably due to myopia and related eye diseases, is a serious health issue that decreases individuals' quality of life and even affects economic and educational opportunities in the modern age of planar displays (1-5). A large number of people of various ages, especially school and university students, have visual deficiencies of differing severities, and the declining trend in visual function appears to be accelerating rapidly with the increased use of flat screens and mobile phones (2). Due to the high incidence of myopia and uncorrected presbyopia $(3,4)$, a fast, convenient, accurate, sensitive, and easily accessible vision assessment and screening technology is needed to determine whether additional steps are necessary in ocular examinations, which are important for preserving visual function and are recommended by the WHO (e.g., the Consultation on Development of Standards for Characterization of Vision Loss and Visual Functioning in 2003) (6).

While a paper eye chart with various optotypes may serve as a simple and effective vision screening method (7), there are obvious drawbacks of this method. First, the functionality of the chart is very simple. Second, the method for collecting assessment data is ineffective and requires supervision during an examination. Third, there is a limited number of test images; consequently, the evaluations of colour blindness, stereo vision, and binocular balance, motivated children are likely to memorize the expected responses - especially during school or kindergarten screenings, when children can communicate with their classmates - which can lead to inaccurate test results. Furthermore, one eye needs to be covered while the other is assessed, which might have an artificial effect on actual visual acuity. Currently, some visual acuity measurement methods and software have been developed, such as the Smartphone-Based Visual Acuity Measurement $(8,9)$, the Electronic Visual Acuity Tester (10), and the AAPOS Vision Screening App (11); however, these focus mainly on visual acuity screening alone. Other visual functions, e.g., stereo vision and binocular balance, cannot be assessed with a simple paper eye chart, and special equipment must be used $(12,13)$; for example, the Bagolini striated lens (14) can be used to examine binocular balance.

Along with the development of $3 \mathrm{D}$ technology, new visual function tests have been developed; these include the TNO stereo test (15), which requires individuals to wear red-green glasses to view left- and right-eye images separately, and the PASS Test 3, the Titmus stereo test (16), the Randot Stereotests, and the Random Dot E Stereotest, which require individuals to wear a pair of polarizing glasses to view images with the each eye separately. The major drawback of these instruments is that they require the use of additional glasses. Therefore, autostereoscopy has attracted a great deal of research and industrial attention in recent years, which has resulted in technologies such as autostereoscopic smartphones based on parallax barrier technology (17) and the Lang Stereotest, which is based on the column-interleaved technique (18).

Notably, the crosstalk and resolution parameters of the display are very important parameters in both visual acuity and visual function tests. Resolution is closely related to the accuracy of a vision test. Due to the limitations of the barrier and lenticular technologies used in traditional autostereoscopic displays, the resolution required for the tests is twice that of the physical pixels in the liquid crystal display (LCD), which means that at least 2 pixels constitute the smallest unit for this type of test. The low resolution can be partially compensated for with $2 \mathrm{~K}$ and $4 \mathrm{~K}$ panels, such as an autostereoscopic smartphone equipped with a $2 \mathrm{~K}$ screen (17). Minimizing crosstalk in an autostereoscopic display is also important to ensure that the left and right eyes can be screened effectively and independently. Certain values of this parameter can not only improve the accuracy of the monocular vision test but also greatly affect the comfort of the testing process. Large crosstalk values might cause severe visual fatigue in some subjects, including some children, causing them to lose interest in autostereoscopy assessments. Therefore, autostereoscopic smartphone is used to evaluate stereoacuity only and cannot reliably measure visual acuity. Furthermore, instruments based on traditional naked-eye 3D technology are prone to inverse apparent phenomena during vision screening; in other words, the display itself can directionally transmit images to the left and right eyes but may not always transmit the images to the appropriate eyes. To address this issue, traditional screening techniques require subjects to close one eye before testing to ensure that the correct picture is 
viewed by the appropriate eye (17), resulting in complex tests that need to be supervised by a professional.

Our laboratory has also begun to develop and evaluate a screening system based on directional backlight autostereoscopy technology. The display can control the amount of crosstalk to be less than 3\% (19-22), which can enable rapid screening assessments for both monocular and binocular vision. Second, the instrument can preserve the resolution of the LCD panel $\left(1920^{*} 1080\right)(19)$, which means that a single pixel is the smallest unit of this test, and the minimum measurable stereo acuity can reach 57 seconds of arc at $1 \mathrm{~m}$, which meets the clinical standard for stereo tests (a threshold value of 60"). With the use of eye-tracking technology in our system, the inverse apparent phenomenon in vision screening can be eliminated, ensuring simplicity and avoiding the need for assistance during the screening process. Furthermore, with mobile units, leap motion, or gesture recognition $(23,24)$, large amounts of data from eye examinations can be rapidly recorded, stored, and retraced using desktops and cloud storage systems.

Can desktop autostereoscopy serve as a tool for vision assessments and screening? Regarding its use in assessments, it offers some apparent advantages, such as not requiring glasses or assistance and being rapid, accurate, and multifunctional. In the present study, the parametric requirement for the desktop autostereoscopic device is discussed, and high-quality directional backlight autostereoscopy is used to conduct the test. The interaction between the examiner and the desktop is described and demonstrated. The assessment's accuracy, repeatability and inter-visit variability values are presented. With this rapid screening process, visual acuity, colour vision deficiency, stereo vision and binocular balance can all be assessed within a few minutes by an examiner. A large amount of the assessment data obtained during vision screening are shown to be valuable for quickly assessing the vision health of a large number of individuals. We present the following article in accordance with the STROBE reporting checklist (available at http://dx.doi.org/10.21037/atm-20-3555).

\section{Methods}

\section{Subjects}

A total of 157 healthy subjects (78 females and 79 males) aged 5-68 $(32.8 \pm 11.7)$ years old were enrolled in this observational, cross-sectional study to compare the clinical repeatability, accuracy, inter-visit variability, likeability and efficiency of the autostereoscopy method and traditional method for determining visual acuity, colour vision, stereo vision, and binocular vision. The study was conducted at the Zhongshan Ophthalmic Center and performed as per the tenets of the Declaration of Helsinki (as revised in 2013). The study protocol was approved by the Zhongshan Ophthalmic Ethical Committee (IRB-ZOC-SYSU) and was also registered at clinicaltrials.gov (PRS, ID NCT03899623). Informed written consent was obtained from all the patients after the nature and aim of the study were explained in detail. The study included healthy individuals older than 18 years of age with a best-corrected visual acuity (BCVA) of <1.0 logarithm of minimum angle of resolution (logMAR) and no ongoing or prior history of ocular or systemic disease.

\section{Autostereoscopy for vision screening}

The autostereoscopic device is equipped with a 24-inch LCD panel, which has a full high-density resolution of $1920^{*} 1080$ pixels. The display density of the screen is 92 PPI. As the LCD signals are sampled at $120 \mathrm{~Hz}$ with a timesequential control synchronized with the backlight, $60 \mathrm{~Hz}$ images or videos viewed by each eye also have $1920^{*} 1080$ pixels. Hence, the minimum pixel size is $0.027 \mathrm{~cm}$, which has a minimum angle of approximately 0.94 ' at $1 \mathrm{~m}$.

The autostereoscopic device is equipped with intelligent eye-tracking cameras to direct the eye chart to the designated eye (Figure 1A). The amount of crosstalk between the left and right channels can be as small as 3\%.

The device is equipped with a mobile phone or a leap motion device to detect gestures (Figure 1B), which is used to record the subject's test results on the desktop and collect the subject's responses to the vision test items. The autostereoscopic and mobile devices can be connected with either Bluetooth or wireless connections, such as $4 \mathrm{G}$ or $5 \mathrm{G}$. The collected data can be received by a desktop system equipped for autostereoscopy. For the convenience of the subject, an app with a user-friendly interface is used to provide instructions on how to use the autostereoscopic device.

For convenient rapid screening, all vision data are obtained with the subject in a particular position away from the autostereoscopic LCD. In this experimental setup, the subject is required to sit in front of the autostereoscopy LCD, which is positioned 1 metre away from the subject (Figure 1B). Eye-tracking technology helps detect the subject's retina for accurate assessments. 
A

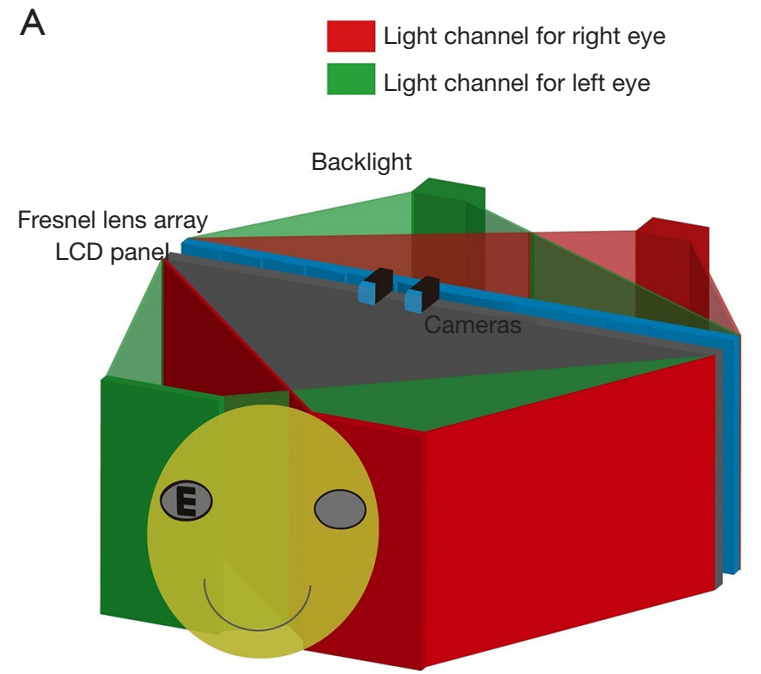

B

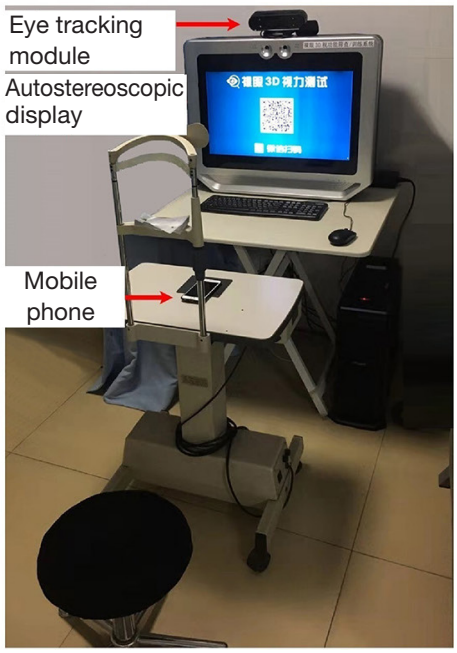

Figure 1 (A) Diagram of the autostereoscopic device with an interactive mobile unit for vision screening. For the test of a single eye, only one channel of the backlight is on to illuminate the tested eye, while the other eye views a white background. For tests of vision parameters related to stereo vision and binocular balance, both channels are on and updated at $60 \mathrm{~Hz}$, and the panel is refreshed at $120 \mathrm{~Hz}$. (B) Picture of the vision screening system. LCD, liquid crystal display.

\section{Visual acuity}

For the accurate measurement of visual acuity, the viewing distance should be accurately specified. In practice, a subject might move from the ideal location, even unintentionally, so the viewing distance must be measured, and the subject must be informed to remain at that distance. For actual measurements, head support will help fix the subject's location. The letter "E" and white blank pictures were displayed on the screen (Figure 2), which could be seen by only a single eye.

The eye chart used in this study was a tumbling $\mathrm{E}$ chart. The letter E, ordered from large to small sizes and rotated by various degrees, appeared randomly on the screen. After viewing the letter, the subject indicated the direction in which the $\mathrm{E}$ was rotated using a wireless mobile unit with "up", "down", "left" and "right" as the inputs, and the inputs were compared with the correct direction. The assessment of visual acuity was terminated when 3 incorrect readings were detected. The results were recorded as $\log$ MAR BCVA.

\section{Colour vision}

The test for colour vision was fairly simple and straightforward. It followed the same procedure as that for the visual acuity assessment. The pattern for colour vision testing adopted in this study is shown in W Figure 3. The results were recorded as normal, anerythropsia, deuteranopia, or protanopia anerythrochloropsia.

\section{Stereo vision}

Stereo vision, or stereopsis, is the coordinated effect of both eyes; therefore, slightly different videos or pictures are received by the left and right eyes to produce $3 \mathrm{D}$ perception. In our initial setup, we used a stationary picture of a 3D object against a flat background (Figure 4). As stereo vision was measured with the image of an object popping out of a similar background, the random dot stereo acuity was quantitatively measured using objects. The objects were a triangle, a circle, and a diamond, and the subjects were asked to indicate which object was hidden the image of random dots. The degree to which the objects popped out of the screen was used to measure stereo acuity. For this paper, the minimum measurable stereo acuity was 57 ". The result was recorded as the stereoacuity.

\section{Binocular balance}

Binocular balance reflects whether each eye plays an equal role in viewing an object. Binocular balance can be measured with a Bagolini striated lens. In this study, the Bagolini striated lens test was well simulated by two 

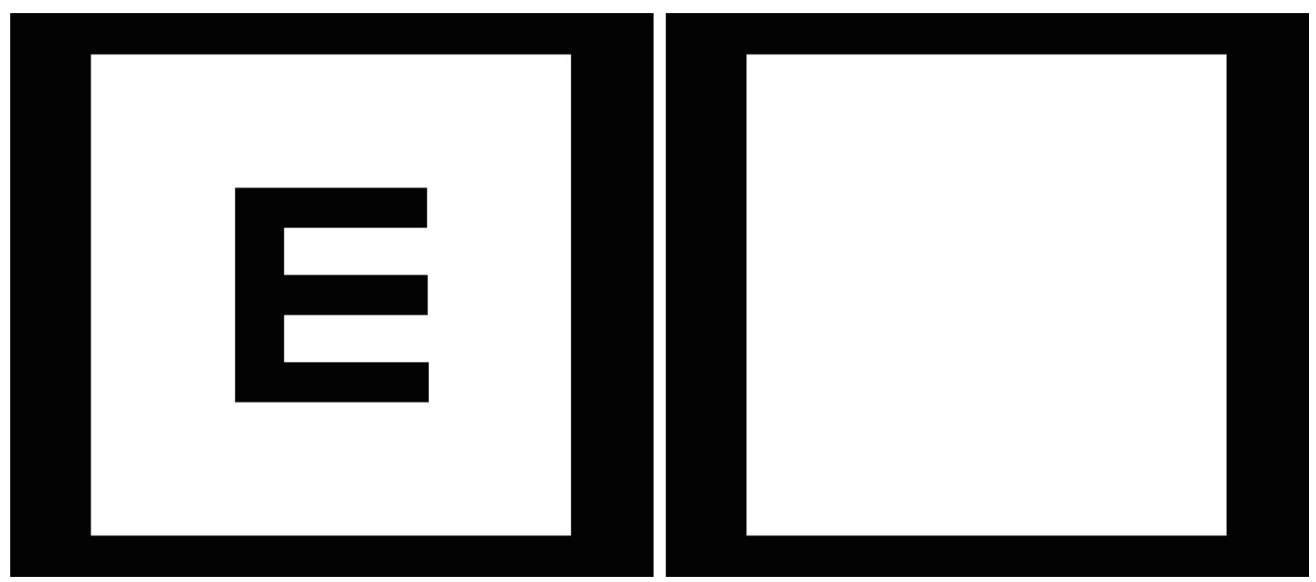

Figure 2 The test configuration and typical left and right patterns used for assessing visual acuity.
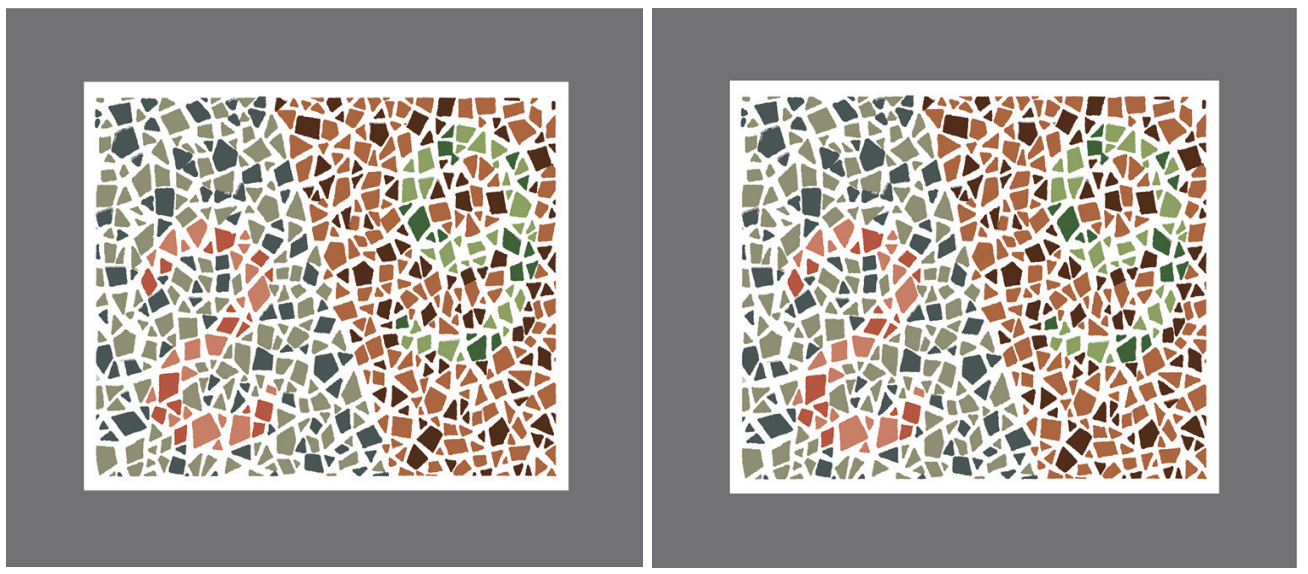

Figure 3 The test configuration and typical left and right patterns used for assessing colour vision.

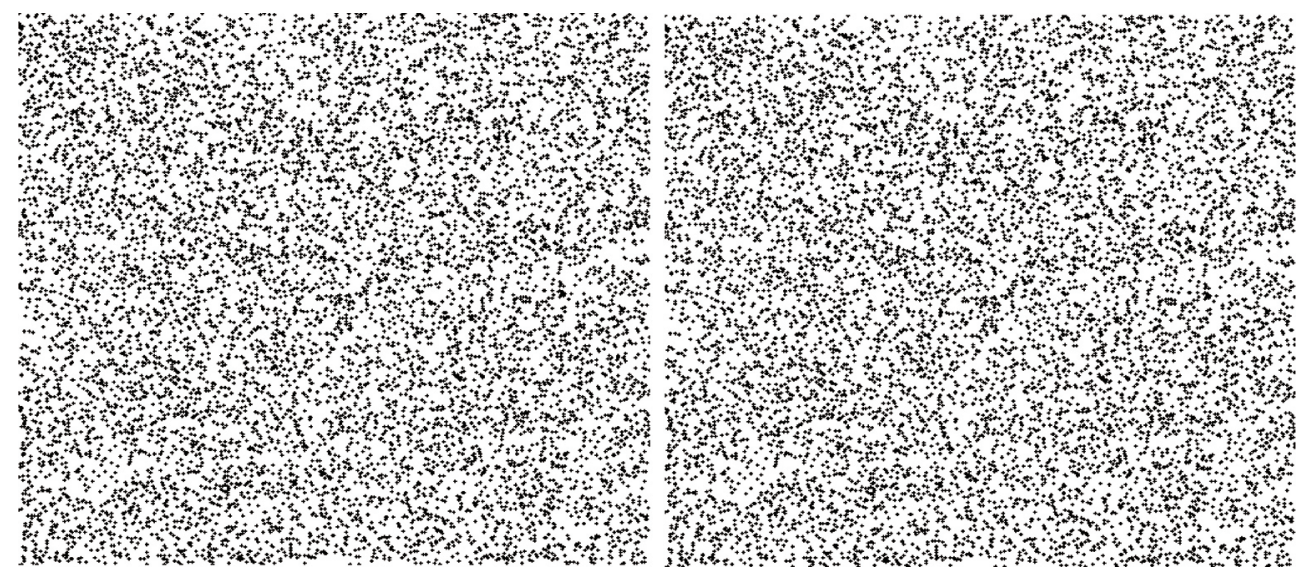

Figure 4 The test configuration and typical left and right patterns used for assessing stereo vision. 

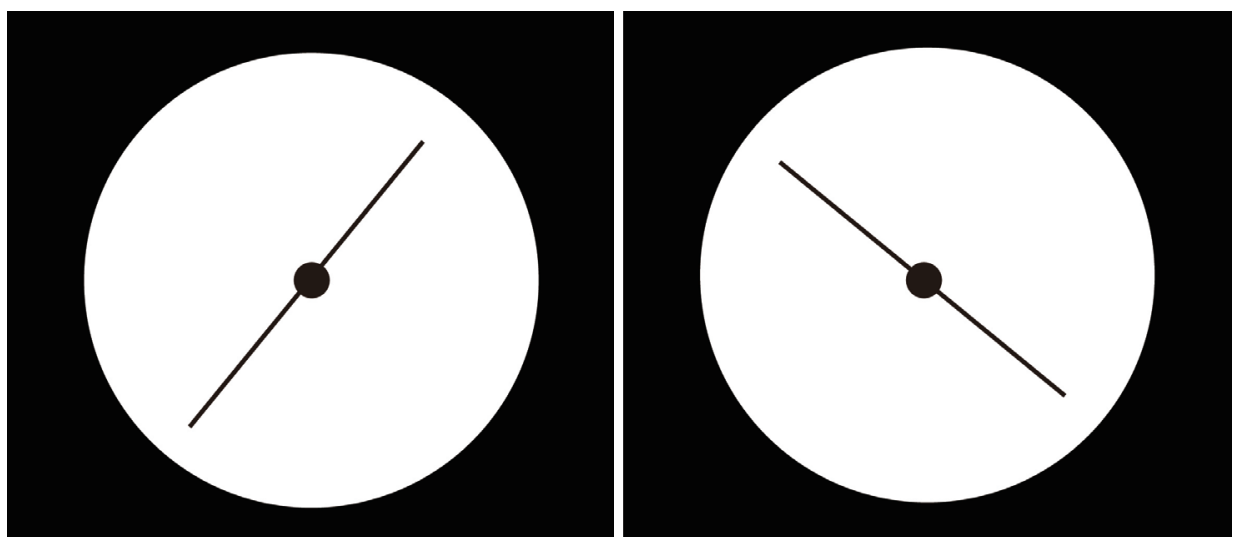

Figure 5 The test configuration and typical left and right patterns used for assessing binocular balance.

pictures, one of which presented a 45-degree diagonal line, while the other presented a 135-degree diagonal line (Figure 5). These two pictures were displayed through the left and right channels. Binocular balance was assessed according to how many lines the subject could see and the degree of the line. The result was recorded as binocular balance or binocular suppression.

\section{Test procedures}

Standardized protocols for data collection, including the training of study personnel, were performed to minimize interobserver variability when multiple individuals were gathering and entering data. The repeatability of the autostereoscopy test was assessed after an interval of 24 hours by a single examiner with recruited participants. Next, the autostereoscopy test and the Early Treatment Diabetic Retinopathy Study (EDTRS) English chart placed at a distance of $5 \mathrm{~m}$ were used to compare the differences in logarithm of the minimum angle of resolution (logMAR) visual acuity. Then, in the interrater reliability test, two testers measured the visual acuity of one subject, and the intraclass correlation (ICC) value was calculated. Moreover, likeability and visual fatigue were assessed with a simple 5 -point grading questionnaire and compared between the EDTRS chart and the autostereoscopy test. Finally, work efficiency was evaluated by calculating the testing time. For the assessment of monocular visual acuity, one channel of the autostereoscopy display was programmed with the appropriate chart or video. For the assessment of binocular vision, the same pictures or videos were sent to both eyes to assess colour vision, while slightly different pictures or videos were sent to both eyes to assess stereo vision and binocular balance.

\section{Statistical analysis}

Statistical analysis was performed using SPSS software, version 22 (IBM, Inc., Armonk, NY). Visual acuity scores were analysed in $\log M A R$. The stereo vision result was recorded as stereoacuity, which had three levels of 63", 100", and 200". Colour vision was recorded as normal, anerythropsia, deuteranopia, or protanopia anerythrochloropsia. Binocular balance was recorded as binocular balance or monocular suppression. Stereo acuity was analysed as an ordinal categorical variable, while colour vision and binocular vision were analysed as unordered categorical variables. The rank-sum test was used to determine the difference in accuracy between the autostereoscopy test and the traditional methods. Limitsof-agreement plots (Bland-Altman plots) were used to assess the difference in measurements between two sessions conducted on different days. A paired $t$-test was used to test the differences in likeability, visual fatigue score and testing time between the autostereoscopy test and the traditional methods. Cases with missing data were deleted. P values less than 0.05 were considered significant.

\section{Results}

A total of 157 healthy subjects (78 females and 79 males) aged 5-68 $(32.8 \pm 11.7)$ years old were enrolled. For the repeatability test, the visual acuity measured with autostereoscopy was $0.045 \pm 0.018$ and $0.035 \pm 0.018$ $(\mathrm{P}=0.702)$ in the first and second tests, respectively (Figure $6 A$ ), and the $95 \%$ limits of agreement spanned from 
A

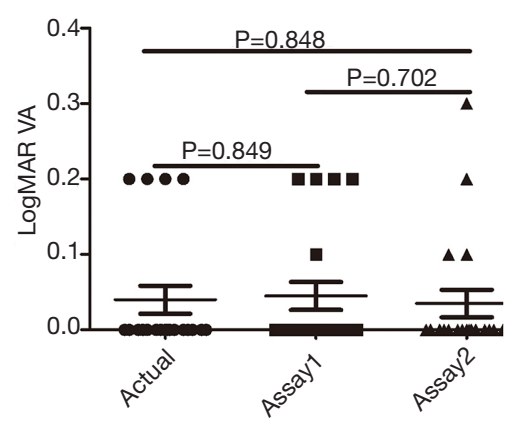

D

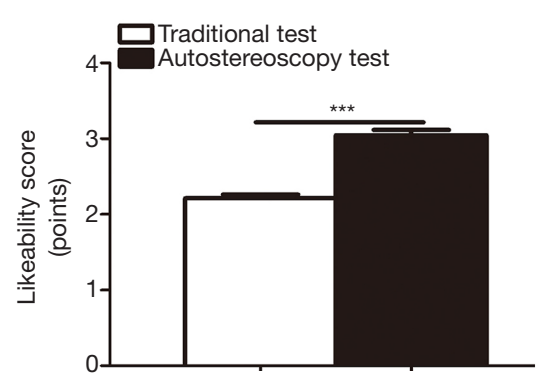

B

E
Bland-altman of VA: difference vs. average
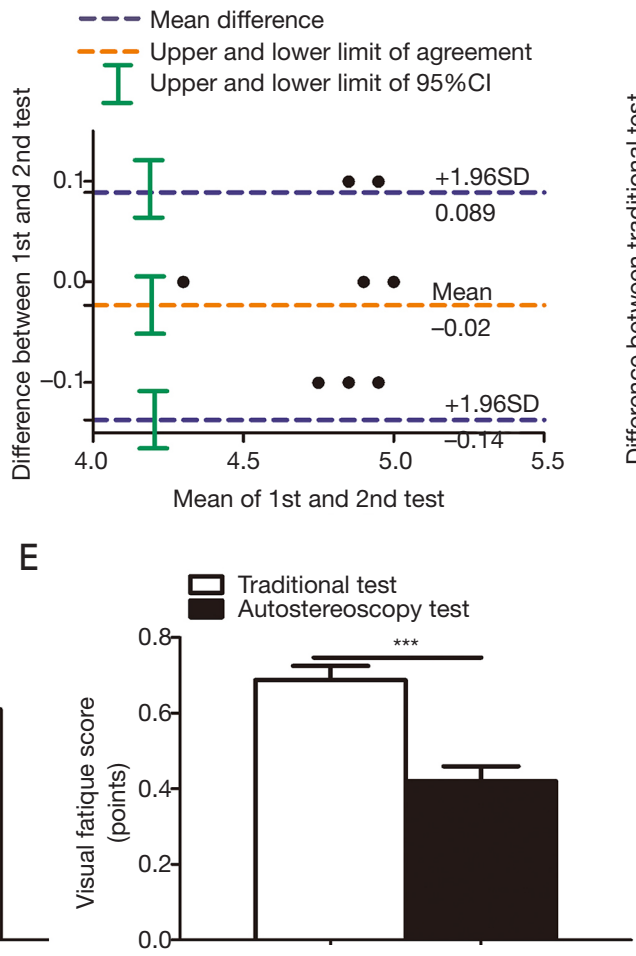

C

Bland-altman of VA: difference vs. average

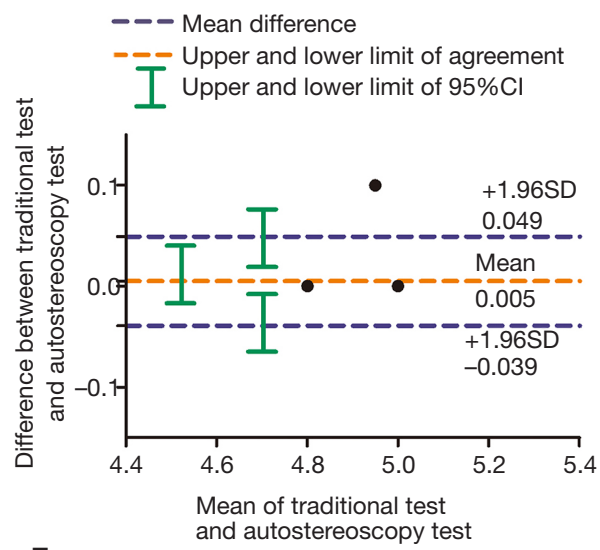

$\mathrm{F}$

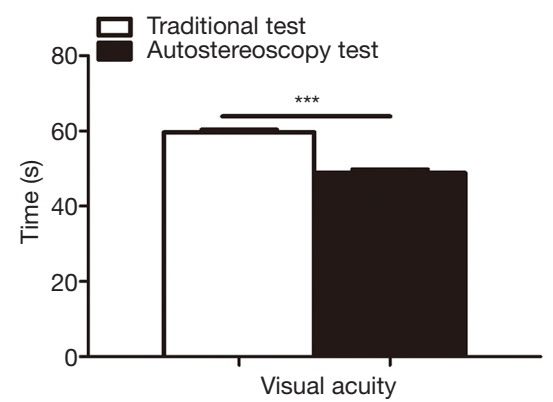

Figure 6 (A)The logarithm of minimum angle of resolution visual acuity (logMAR VA) for the Early Treatment Diabetic Retinopathy Study (EDTRS) chart (Actual) and the autostereoscopy test (Test 1 and Test 2); (B) Bland-Altman plots for the comparison of the $1^{\text {st }}$ and $2^{\text {nd }}$ autostereoscopy tests; (C) Bland-Altman plots for the comparison between the autostereoscopy test and EDTRS chart; (D) the likeability scores for the traditional test and the autostereoscopy test; (E) the visual fatigue scores for the traditional test and the autostereoscopy test; (F) the working time for the traditional test and autostereoscopy test.

-0.14 to 0.09 for $\log$ MAR visual acuity, which was not significantly different from 0 (Figure $6 B$ ). The colour vision, stereo vision, and binocular vision results were qualitative and were the same for the autostereoscopy test and the traditional methods.

The mean $\log$ MAR visual acuities measured with the EDTRS chart and the autostereoscopy test were $0.04 \pm 0.02$ and $0.05 \pm 0.02$, respectively, which were not significantly different $(\mathrm{P}=0.849)$, and the $95 \%$ limits of agreement spanned from -0.039 to 0.049 for $\log$ MAR visual acuity, which was not significantly different from 0 (Figure 6C). The correlation between these two kinds of tests was statistically significant (Spearman correlation coefficient $=0.829, \mathrm{P}<0.001)$. The median $(25 \%, 75 \%)$ stereo vision measured with the random dot $3 \mathrm{~S}$ stereo acuity test and the autostereoscopy test were 63" (63", 63") and 63" (63", 63 "), respectively, which were not significantly different
( $\mathrm{P}=0.411)$. The stereo test had three levels of 63 ", 100", and $200 "$. The proportions were $95.8 \%, 2.5 \%$, and $1.7 \%$ for the random dot $3 \mathrm{~S}$ stereo acuity test and $92.4 \%, 3.7 \%$, and $3.8 \%$ for the autostereoscopy test, respectively, and the coincidence rate was $93.83 \%$. The correlation between the two kinds of tests was statistically significant (Spearman correlation coefficient $=0.829, \mathrm{P}<0.001$ ).

Regarding likeability, the EDTRS chart and autostereoscopy test had scores of $2.21 \pm 0.53$ and $3.04 \pm 0.07$, respectively $(\mathrm{P}<0.001)$, while the scores for colour vision, stereo vision, and binocular vision with the traditional test and the autostereoscopy test were $2.02 \pm 0.59$ and $3.36 \pm 0.93$, respectively $(\mathrm{P}<0.001)$, indicating that the autostereoscopy test was more popular (Figure 6D).

For the visual fatigue evaluation, the mean visual fatigue scores were $0.69 \pm 0.04$ and $0.42 \pm 0.04(\mathrm{P}<0.001)$ for the EDTRS chart and autostereoscopy test, with scores of 
$3.95 \pm 0.30$ and $1.51 \pm 0.50(\mathrm{P}<0.001)$ for colour vision, stereo vision, and binocular vision, respectively, indicating that the latter test did not induce considerable visual fatigue (Figure 6E).

Regarding work efficiency, the testing times were $59.65 \pm 0.66$ and $48.92 \pm 0.86 \mathrm{~s}(\mathrm{P}<0.001)$ for the EDTRS chart and autostereoscopy test, respectively. For colour vision, stereo vision, and binocular vision, the testing times were $378.49 \pm 144.48$ and $251.36 \pm 97.74 \mathrm{~s}(\mathrm{P}<0.001)$ for the traditional test and the autostereoscopy test, respectively (Figure 6F).

The autostereoscopy test was conclusively shown to be a valid, efficient and repeatable tool for the measurement of colour vision, stereo vision, and binocular vision, and the process was found to be subjectively well liked and comfortable.

\section{Discussion}

There are a variety of standard ophthalmic examinations that can be performed in the clinic; however, most of them are paper tests, and the methods for collecting the assessment data are ineffective $(17,25)$. The functionality of these examinations is very simple; however, functions such as stereo vision and binocular balance cannot be assessed with a simple paper eye chart, and special equipment must be used to assess different visual functions. For the stereo vision test, polarizing glasses and red-green glasses can be used to assess one eye at a time on the monocular test without additional occluding plates, and the use of glasses might have an artificial effect on actual visual acuity. Autostereoscopic displays send separate images to the left and right eyes so that viewers can perceive $3 \mathrm{D}$ effects without wearing assistive glasses.

The conventional autostereoscopic display consists of either a lenticular lens array (26) or a barrier array (27) to deliver different images to each eye. These two methods have two common drawbacks: reduced resolution and a large amount of crosstalk $(28,29)$. The display resolution is directly related to visual acuity. It should be noted that the actual resolution should be determined by the examined eye rather than by screen resolution, which depends on technical aspects of the autostereoscopic display used in the test. On the other hand, minimizing the crosstalk between the left and right channels of an autostereoscopic device is very important to guarantee that vision screening can be effectively and independently conducted for the left and right eyes (7). Furthermore, large amounts of crosstalk might cause severe visual fatigue in some subjects, and it is likely that some subjects, including some children, might reject the use of autostereoscopy for assessments. The autostereoscopic device used in this experiment adopted directional backlight technology, which can control the amount of crosstalk to less than $3 \%$. Moreover, with the interactive connection of the autostereoscopic device with a mobile unit or gesture recognition software, massive amounts of data from eye examinations can be rapidly recorded, stored and retraced from desktop and cloud storage systems.

Usually, monocular vision screening assessments are performed by sending a programmed standard eye chart into the right channel, which is received by the designated eye. To reduce the artefacts arising from crosstalk, a programmed white blank picture is sent to the other eye so that possible crosstalk will be immersed by the large background and will not be visible to the wrong eye. This method has been shown to be effective in eliminating residual optotypes, even though the other eye is not covered at any time during the assessment. On the other hand, for the assessment of binocular visual function, such as stereo vision and binocular balance, two different pictures or videos are sent to both eyes to produce a sense of depth related to $3 \mathrm{D}$ perception.

Notably, the eye-tracking technique is very important; although subjects are required to sit without moving, their retinas might still move around, which would affect the test results. To eliminate the assessment uncertainties caused by the subject's movement, an eye-tracking technique was applied. With this technique, two front cameras were used to determine the exact location of the retina, and the test pictures or videos were sent to the subject's eyes to detect any possible movement, hence enhancing the accuracy of the examination. Furthermore, the eye-tracking technique can be used to instantaneously detect a subject's movement using state-of-the-art eye-tracking technique with motion prediction to produce a flicker-free viewing experience $(30,31)$.

In the current study, the repeatability, accuracy, and intervisit variability were good, and there was a good correlation between the autostereoscopy assessment and the traditional test, which suggests that the autostereoscopy assessment can accurately assess visual acuity. Moreover, the mean difference between the autostereoscopy assessment and the traditional test was not statistically significant. Although visual acuity screening is often limited by issues of recognition and resolution acuity, this study found that the autostereoscopy 
assessment could be performed in individuals as young as 5 years old. Despite its inherent limitation as a subjective screening device that requires feedback from the examinee, the autostereoscopy assessment has advantages over other vision screening modalities. One advantage is that it does not require individuals to cover one eye, so the assessment is sanitary, fast, efficient and convenient, and the testing time was faster than that of the traditional test. Another advantage is that it uses interactive software that increases interest, which is beneficial for its clinical application and popularization; thus, it was more popular among the subjects than the traditional test. The interactive software is fast, convenient and sanitary, and it has the potential to assess a subject's colour vision, stereo vision and binocular balance within a few minutes. Moreover, it should be noted that future assessments can be conducted with a desktop computer with a high-resolution PPI screen, resulting in even higher accuracy for various applications.

\section{Conclusions}

Autostereoscopy assessment was shown to be reproducible, accurate, subjectively well-liked and comfortable and fast, and it is expected to be used widely in clinics.

\section{Acknowledgments}

Funding: This work was supported by the Key Area Research and Development Program of Guangdong Province (No. 2019B010152001) and the National Science Foundation of China (No. 11534017, No. 61991452).

\section{Footnote}

Reporting Checklist: The authors have completed the STROBE reporting checklist. Available at http://dx.doi. org/10.21037/atm-20-3555.

Data Sharing Statement: Available at http://dx.doi. org/10.21037/atm-20-3555. The authors confirm that the data supporting the findings of this study were made available from a third-party network disk immediately upon publication.

Peer Review File: Available at http://dx.doi.org/10.21037/ atm-20-3555

Conflicts of Interest: All authors have completed the ICMJE uniform disclosure form (available at http://dx.doi. org/10.21037/atm-20-3555). The authors have no conflicts of interest to declare.

Ethical Statement: The authors are accountable for all aspects of the work and for ensuring that questions related to the accuracy or integrity of any part of the work are appropriately investigated and resolved. The study was conducted in accordance with the Declaration of Helsinki (as revised in 2013). The study was approved by the institutional review board of Zhongshan Ophthalmic Centre of Sun Yat-sen University (IRB-ZOC-SYSU). All procedures performed in studies involving human participants were in accordance with the ethical standards of the institutional and/or national research committee(s). Written informed consent was obtained from the patients for the publication of this study and any accompanying images. A copy of the written consent form is available for review by the editor-in-chief of this journal.

Open Access Statement: This is an Open Access article distributed in accordance with the Creative Commons Attribution-NonCommercial-NoDerivs 4.0 International License (CC BY-NC-ND 4.0), which permits the noncommercial replication and distribution of the article with the strict proviso that no changes or edits are made and the original work is properly cited (including links to both the formal publication through the relevant DOI and the license). See: https://creativecommons.org/licenses/by-nc-nd/4.0/.

\section{References}

1. Bourne RRA, Flaxman SR, Braithwaite T, et al. Magnitude, temporal trends, and projections of the global prevalence of blindness and distance and near vision impairment: a systematic review and meta-analysis. Lancet Glob Health 2017;5:e888-97.

2. Zhang X, Wang Y, Huang D, et al. Prevalence of reduced visual acuity among preschool children in eastern China and comparison at a 5-year interval. Clin Exp Ophthalmol 2018;46:994-1001.

3. Foreman J, Keel S, van Wijngaarden P, et al. Prevalence and causes of visual loss among the indigenous peoples of the world: a systematic review. JAMA Ophthalmol 2018;136:567-80.

4. Delcourt C, Le Goff M, von Hanno T, et al. The decreasing prevalence of nonrefractive visual impairment in older europeans: a meta-analysis of published and 


\section{Page 10 of 10}

unpublished data. Ophthalmology 2018;125:1149-59.

5. He M, Xiang F, Zeng Y, et al. Effect of time spent outdoors at school on the development of myopia among children in China: a randomized clinical trial. JAMA 2015;314:1142-8.

6. Jonas DE, Amick HR, Wallace IF, et al. Vision screening in children aged 6 months to 5 years: evidence report and systematic review for the US preventive services task force. JAMA 2017;318:845-58.

7. Zhao L, Stinnett SS, Prakalapakorn SG. Visual acuity assessment and vision screening using a novel smartphone application. J Pediatr 2019;213:203-10.e1.

8. Brady CJ, Eghrari AO, Labrique AB. Smartphone-based visual acuity measurement for screening and clinical assessment. JAMA 2015;314:2682-3.

9. Rono HK, Bastawrous A, Macleod D, et al. Smartphonebased screening for visual impairment in Kenyan school children: a cluster randomised controlled trial. Lancet Glob Health 2018;6:e924-32.

10. Kupl MT, Dobson V, Peskin E, et al. The electronic visual acuity tester: testability in preschool children. Optom Vis Sci 2004;81:238-44.

11. Nik Azis NN, Chew FLM, Rosland SF, et al. Parents' performance using the AAPOS vision screening app to test visual acuity in Malaysian preschoolers. J AAPOS 2019;23:268.e1-e6.

12. Cooper J, Feldman J, Medlin D. Comparing stereoscopic performance of children using the Titmus, TNO, and Randot stereo tests. J Am Optom Assoc 1979;50:821-5.

13. Nabie R, Andalib D, Khojasteh H, et al. Comparison of the effect of different types of experimental anisometropia on stereopsis measured with Titmus, Randot and TNO Stereotests. J Ophthalmic Vis Res 2019;14:48-51.

14. Hirai T, Arai M, Ito Y, et al. Modified Bagolini striated glass test: clinical applications of starlight test in binocular visual field screening. Br J Ophthalmol 1998;82:1288-93.

15. Lee JY, Seo JY, Baek SU. The effects of glasses for anisometropia on stereopsis. Am J Ophthalmol 2013;156:1261-6.e1.

16. Tejedor J, Ogallar C. Comparative efficacy of penalization methods in moderate to mild amblyopia. Am J Ophthalmol 2008;145:562-9.

17. Zhao L, Wu H. Stereoacuity measurement using an autostereoscopic smartphone. Ann Transl Med 2019;7:390.

18. Lang J. A new stereotest. J Pediatr Ophthalmol Strabismus 1983;20:72-4.

19. Fan H, Zhou Y, Wang J, et al. Full resolution, low crosstalk, and wide viewing angle auto-stereoscopic display
Li et al. An interactive desktop autostereoscopy vision assessment

with a hybrid spatial-temporal control using free-form surface backlight unit. J Disp Technol 2015;11:620-4.

20. Liang H, An S, Wang J, et al. Optimizing timemultiplexing auto-stereoscopic displays with a genetic algorithm. J Disp Technol 2014;10:695-9.

21. Wang J, Liang H, Fan H, et al. High-quality autostereoscopic display with spatial and sequential hybrid control. Appl Opt 2013;52:8549-53.

22. Krebs $\mathrm{P}$, Liang H, Fan H, et al. Homogeneous freeform directional backlight for 3D display. Opt Commun 2017;397:112-7.

23. Li X, Chen CH, Huang YP. 3D interactive system based on vision computing of direct-flective cameras. J Soc Inf Disp 2016;24:521-8.

24. Kumar P, Saini R, Behera SK, et al. Real-time recognition of sign language gestures and air-writing using leap motion. In: 2017 fifteenth IAPR international conference on machine vision applications (MVA). Nagoya, Japan: IEEE; 2017:157-160.

25. Budai A, Czigler A, Mikó-Baráth E, et al. Validation of dynamic random dot stereotests in pediatric vision screening. Graefes Arch Clin Exp Ophthalmol 2019;257:413-23.

26. Zhao WX, Wang QH, Wang AH, et al. Autostereoscopic display based on two-layer lenticular lenses. Opt Lett 2010;35:4127-9.

27. Lv GJ, Wang QH, Zhao WX, et al. 3D display based on parallax barrier with multiview zones. Appl Opt 2014;53:1339-42.

28. Lee J, Kim J, Koo G, et al. Analysis and reduction of crosstalk in the liquid lenticular lens array. IEEE Photonics J 2017;9:2900108.

29. Shin D, Kim J, Kim C, et al. 3-D image crosstalk reduction by controlling the width of the electrode in a liquid lenticular lens. IEEE Photonics J 2018;10:7000412.

30. Zhang H, Chen M, Li X, et al. Overcoming latency with motion prediction in directional autostereoscopic displays. J Soc Inf Disp 2020;28:252-61.

31. Chen M, Li X, Zhang H, et al. Quantitative measurement and reduction of flicker in directional backlight autostereoscopic displays. Opt Commun 2020;458:124714.

Cite this article as: $\mathrm{Li} \mathrm{X}$, Zhong J, Wang Y, Zhang H, Li J, Li K, Gu L, Zheng M, Yuan J, Fan H, Deng D, Wang Y, Zhou J. Rapid, accurate, multifunctional and selfassisted vision assessment and screening with interactive desktop autostereoscopy. Ann Transl Med 2021;9(1):23. doi: 10.21037/atm-20-3555 